This whole subject is an old one and has been referred to by many writers on pneumonia, but it is frequently overlooked. I did not intend to take a crack at the surgeons, but as Dr. McCrae has opened up this line of thought I may say that in talking on this topic of referred abdominal pain with a couple of surgeons one said he had never heard of such a thing and the other said he did not believe it ever occurred. It was largely because of this conversation that I thought a paper on the subject might be timely and perhaps instructive.

\section{THE PASSING OF CHRONIC RHEUMATISM.*}

JAMES J. WALSH, M.D., PH.D.

Lecturer on General Medicine at the N. Y. Polyclinic School for Graduates in Medicine. NEW YORK.

In response to the question, "Have you ever suffered from rheumatism?" addressed to the ordinary patient over 40 years of age, the physician seldom gets a negative answer. Even younger patients will tell of having had vague pains in their arms or shoulders, or in their knees or ankles, that they consider to have been rheumatic. Popularly, the notion is that rheumatism is one of the ills to which flesh is almost inevitably heir, and, in the course of time, practically every one is supposed to have some touches of it. It is not too much to say, then, that rheumatism is popularly considered to be practically. a universal disease. Physicians encouraged this idea, to a certain extent at least, by suggesting that vague pains are rheumatic in origin, or at least agrceing with patients when they say that probably they have rheumatism.

As a matter of fact, many of the irregular practitioners in recent years, particularly the osteopaths, draw their clientele (and it is by no means innumerous), from the ranks of the so-called chronic rheumatics. Most of the proprietary medicines, besides their other wonderworking effects, are advertised to be good for chronic rheumatism, and especially for that fateful condition, the uric acid diathesis, which is supposed to be provocative of chronic aches and pains of many kinds.

To talk, then, of the passing of chronic rheumatism in the sense of the gradual disappearance of the affection during recent years, would seem to be highly paradoxical. Never, more than at the present time, did patients insist on treatment for the relief of pains and aches supposed to be due to chronic rheumatic conditions of one kind or another. That there are many symptom complexes to which the name rheumatism is commonly applied is beyond doubt. How many of these pathologic processes have anything in their underlying basis of causation to justify the name rheumatism is a very doubtful question.

Chronic rheumatism would supposedly be a condition in which some of the pathologic conditions of acute rheumatism persisted, and in which, as a consequence of this persistence, tissue changes of various kinds took place, causing a deterioration of joint structures. As a matter of fact, however, it will be found that while very many patients confess to chronic rheumatism, comparatively few will admit having suffered from the acutely painful red swollen joint, accompanied by fever causing confinement to bed, which characterizes true acute rheumatism. It is now generally conceded that acute articular rheumatism is a specific infectious disease, and while other specific infections, as, for instance, gonorrhea, influenza or typhoid fever, may produce disorganizing

* Read at the Fifty-fourth Annual Session of the American Medical Association, in the Section on Practice of Medicine, and approved for publication by the Executive
Anders, Frank A. Jones and W. S. Thayer. conditions in joints, it is a typical sign of acute rheumatic arthritis to go on to complete recovery. When changes in joints remain after acute rheumatism there is always some suspicion of the possibility of secondary infection, or even a doubt as to the original diagnosis, acute articular rheumatism having been the proper one.

A little examination of the cases commonly called chronic rheumatism shows how many disparate conditions are jumbled together under this term. Vague pains of any kind, for which no direct cause can easily be found, are at once called chronic rheumatism. Many of these conditions can be traced to definite nervous and mechanical conditions without much difficulty if careful examination of each individual case be made. I have already pointed out in previous papers that there are at least fourteen different achy conditions which are often called chronic rheumatism, but without any good reason. The most common form of pseudo-rheumatism is the relaxation of joints which occurs in the ankle and is known as flat-foot, or which follows the dislocations of joints producing tissue conditions which make the patients very sensitive for all their after-life to changes in the weather. Just as there are relaxations at the ankle joint, so there may be relaxations of other jointsthe knee, the hip and the shoulder-which are apt to cause something of the muscular discomfort that becomes so prominent a symptom of flat-foot, or the use of dislocated joints in which relaxation has taken place.

A second very common form of vague pain usually attributed to rheumatism, occurs in connection with occupation neuroses. There is not a single occupation in which some movement involving the co-ordination of a number of muscles has to be made and frequently repeated, that may not be the source of pains and spasmodic inability to use muscles properly, such as occurs so commonly in connection with writer's cramp, or telegrapher's spasm. These movements need not necessarily be very complicated in order to have this discomfort develop. The lower limbs may be the subject of these occupation neuroses, as in the cases of men who work foot-power presses, or in those who run sewing machines, or even in nervous individuals who have to sweep much and constantly use the broom from one side supporting the weight on one leg, while the sweeping movement is accomplished with the arm. Filers, carpenters, laundresses who iron much, planers, men who have to use hammer and chisel, all suffer from these neurotic conditions, usually more painful on rainy days, and for this reason so commonly called rheumatism.

A third source of vague pains that may, if not carefully investigated, be attributed to rheumatism is neuritis. In persons who are exposed to the weather and who use one set of muscles much more than others, especially if at the same time there is the absorption into the system of such toxic substances as lead or alcohol, there not infrequently develops a neuritis of the nerve supplying the overworked group of muscles. Stonecutters, for instance, who use their deitoid and other shoulder girdle muscles for the purpose of frequently lifting a heavy hammer or mallet, and whose exposure to the elements leads them to take alcohol often in greater quantities than is good for them, develop a neuritis of that branch of the circumflex nerve which supplies these muscles. At first this makes the muscles much more easily tired than before. During this period other muscles in the neighborhood are asked to do more work than usual in order to make up for the paretic muscles. As a consequence, painful, overfatigued conditions develop. After a while, the neuritis continuing, there is 
almost complete loss of power in the deltoid, and then the man can no longer pursue his occupation.

It must be remembered that the branch of the circumflex nerve which supplies the deltoid muscle at the shoulder also sends certain filaments to the joint structures of the shoulder. These filaments are really trophic fibers, and as a consequence the neuritis set up in them causes a lowering of the nutrition of the joint structures. This, together with the forced disuse owing to the inability to use and co-ordinate the muscles of the shoulder girdle, leads to something resembling degenerative changes within the joints. Points of tenderness develop especially over the head of the humerus, and the absence of synovial fluid may lead to crackling and roughness, so that there is some grating or fremitus when passive movements of the joints are made. It is easy to attribute such conditions to chronic rheumatism and to consider the neuritis in the case as rheumatic in origin and perhaps secondary to the joint changes, if the examiner does not follow out the history of the case carefully and examine what was the real beginning of the pathologic condition.

Some of these neuritic cases run a very insidious course, and it may not be clear for some time what the condition actually is. A very characteristic illustrative case of this kind has been recently under observation. The patient was a painter who did inside work, being occupied mainly with the painting of balustrades, doors, window-frames, and picture-moldings, requiring him frequently to occupy awkward positions and use many movements of the wrist in painting small carved portions of woodwork. He came complaining of vague pains in the forearm, and occasionally in the shoulder, which were worse on rainy days. He had been a painter for nearly ten years, and had never suffered from any symptoms of lead poisoning. There was no blue line on his gums. I concluded that it was a simple occupation neurosis, since the tired feelings that he described, and the tendency not to be able to work after a while, and the fact that the use of the brush in his left hand for a time had caused the development of the same condition, to a slight extent at least, in the other arm, were characteristic of such ailments.

One day he came to be treated for a sprained wrist. He had fallen on his right hand, and there was some effusion into the joints of the wrist. Two days later he appeared with a characteristic drop wrist. This had developed during the night, and his observation with regard to other painters rather led him to suspect what the true nature of the condition was. It is not unusual for drop wrist to develop after some injury to the part, just as it is quite a common story to have the first symptoms of toe drop as a result of alcoholic neuritis follow upon a sprain of the ankle. In both cases it is evident that for some time, probably for months, there has been a slow running, low grade and practically latent neuritis. It can easily be understood, however, how the preliminary manifestations of this would be a fatigue of muscles coming on much easier than before the nervous affection began, and a tendency to neurotic conditions such as occur in occupation neuroses because of the difficulty of co-ordinating complex muscle movements when the nerve paths leading to the group of muscles are disturbed by the presence of inflammatory products.

A certain number of cases of so-called chronic rheumatism here in America which present evidences of degeneration in joint structures following acute attacks of arthritis, are undoubtedly not genuinely rheumatic in origin, but are of gouty nature. Gout is much more frequent in this country than has been considered to be the case. Ordinarily gout is considered an English disease, and we look for English heredity in those in whom we suspect its presence. The absence of such heredity is, in many physicians' minds, an argument against an affection being gouty. Ordinarily, it would be considered, I suppose, not too much to say that gout is at least four or five times more frequent in England than it is in America. As a matter of fact, the comparison of the statistics of patients admitted to Johns Hopkins Hospital in Baltimore suffering from gout with those admitted to St. Bartholomew's Hospital in London for the same affection are in the proportion of only three to four. That is, gout is three-fourths as frequent in this country as it is in England. How difficult it is at times to differentiate acute gout from acute rheumatism may be judged from the confessions of excellent clinical observers, including Professor Osler himself, that only after two or three attacks of supposed acute rheumatism have occurred in a series of years in patients under their observation did they find the gouty nodules in the ears or the tophi in the fingers which proved that former attacks had been gout, and not rheumatism.

Gout is, in this country, earned rather than inherited. It occurs especially among those who are subject to the influence of lead and those who indulge too freely in malt liquors. While our beer is much less likely to cause the condition than the heavier malt liquors of England, there is now no doubt that the increased consumption of even light beer which has occurred in recent years in this country have made many more people liable to gouty attacks than before. As to lead poisoning, it must not be forgotten that besides painters and workers in lead factories, plumbers, typesetters, stereotypers and others engaged in the printing trades are likely to suffer from lead poisoning if they are susceptible to plumbum. The accumulation of lead in the tissues always predisposes to gout and leads eventually to changes in joints which may be described as chronic rheumatism if the real nature of the pathologic condition and its true etiology are not recognized by careful elicitation of the history of the case.

In recent years the condition known as rheumatic gout, or rheumatoid arthritis, or, better than any of these names, since it does not assume a knowledge of the etiology of the condition of which we have not yet described well, arthritis deformans, has occupied much more attention. We now know that this condition may occur in three forms. There is the slow running form, which begins usually as Heberden's nodes, and sometimes, though not always, goes on to involve other joints besides the terminal joints of the fingers, and so causes deforming, disabling deformity. The pathologic basis of this is a true productive osteitis of the ends of the bones near the joint surfaces, which seems to be due to the presence of irritants in the blood. It occurs by preference in nervous people. The acute attack of pain and discomfort in the affected joints, which are noted especially at the beginning of the affection, are likely to be more frequent and more annoying whenever the patient is under emotional stress or suffering from worry for any reason.

The second form of rheumatoid arthritis is usually called osteoarthritis and in contradistinction to the first it occurs more commonly in men than in women; it is seen by far most frequently in elderly male subjects. It 
consists of a true osteoarthritis usually confined to the hip joint and spoken of by orthopedists not infrequently as senile coxitis. It may occur also in the joints of the spinal column, producing a certain amount of soldering together of the vertebre and consequent fixation of the back, which gives a very characteristic gait. Like the affection of a single large joint, this spondylitis deformans may be absolutely confined, as its name indicates, to the vertebral column, and is, in fact, usually so confined. This form of the arthritis deformans may occur in rounger subjects than the coxitis deformans already referred to.

Neither of the two forms of rheumatoid arthritis described are likely to be taken for rheunnatism by a carefully observant practitioner, though very few patients suffering with these affections come under treatment without being convinced that they are suffering from an affection of rheumatic origin. The third form of rheumatoid arthritis, however, has many symptoms in common with rheumatism, and is undoubtedly one of the main reasons why acute rheumatism is thought to produce chronie pathologic conditions in joints oftener than it really does. This third form of arthritis deformans, called by English observers acute rheumatoid arthritis, is described very well by $W$. Hale White in the last number of Guy's Hospital Report, Vol, 57, 1902.

This third form of arthritis deformans occurs for the most part in women whose age is usually not far from 20 . The disease begins, or is at any rate most prominent, in the proximal phalangeal joints, differing in this from the slow-running form of arthritis deformans which in elderly women begins in the distal phalangeal joints. Acute rheumatoid arthritis is usually strikingly seen in the wrists. It is markedly symmetrical, as a rule, in both hands. 'The swelling is considerable, and extends beyond the affected joints, so that there is a distinct fusiform swelling about the interphalangeal articulations and a general swelling about the wrist and other joints. Sometimes a creaking may be felt, due apparently to thickening of the synovial membranes. In the course of a very few weeks, as a rule, most of the joints in the body are affected, so that the patient becomes bedfast, and is unable to move fingers, thumbs or wrists, and sometimes not the elbows, shoulders nor even any of the joints of the lower extremities. The joints of "the spine are often affected, so that turning in bed is difficult and painful, and the temporomaxillary joint is frequently implicated, so that mastication becomes a matter of great discomfort.

Muscular wasting is so very rapid that it is clearly an extreme degree of genuine arthritic atrophy not dependent on disuse. The wasting is usually seen best in the hand and forearm, but in a bad case all the muscles may become distinctly atrophic. The most interesting thing about the disease is the temperature. There is a daily rise toward evening up to $101 \mathrm{~F}$. or $102 \mathrm{~F}$. Exposed parts of the patient are likely to be covered with sweat, and, as can be readily understood, there is strong simulation of acute articular rheumatism. The points of differentiation from articular rheumatism are quite clear, however. The disease begins in the fingers, gradually affects all the other joints, seldom fails to involve the temporomaxillary joint, and very commonly affects the joints of the spine, which are seldom attacked by true acute rheumatism. The disease runs a definite course, the temperature coming to the normal after three to four weeks. Much immobility and some swelling around the joints remain. Most patients have a distinct tendency to relapses during which the joints are once more swollen and tender as before. After the febrile stage is passed swelling of the synovial membranes and the soft tissues around the joints remains and the muscular atrophy causes disability. This condition may not improve much, and the patient may remain a chronic invalid.

It is easy to understand how cases of this kind, which have been described by such excellent observers as Garrod and Bannatyne, as well as Hale White in England, would give the impression of an acute rheumatism running over into chronic rheumatism. This form of arthritis deformans is, however, a disease by itself, usually following some infectious fever, as influenza, parotitis, tonsillitis or the like, and perhaps itself of specific microbic origin.

It is easy to understand, from all that has been said, what little space is left for so-called chronic rheumatism. There would seem to be a few cases of true acute rheumatic arthritis, or of the subacute variety which produce changes in the joint tissues that are persistent and progressive. There is a growing impression that even of these few cases the majority are due not to rheumatism alone, but a mixed infection, the secondary infection being responsible for the persistent pathologic lesions. Certainly cases of true chronic rheumatism, that is of progressive pathologic changes in joint tissues for which no other cause except rheumatism can be found, are very rare. So much so, very probably, as to constitute them a medical curiosity. Personally, I should like very much to see such a case, for I am free to confess that I have not as yet seen one.

Is it possible, then, that chronic rheumatism is to be discussed without even a mention of the uric acid diathesis. Personally, I see no reason for hauling in a diathesis for which we have no substantial proof. Certainly the vague pains that are usually attributed to the uric acid diathesis or to lithemia are, in my experience, due more to a neurotic constitution as a predisposing factor and overwork of particular groups of muscles usually under unfavorable conditions as a direct etiologic agent, than to any other cause.

This, of course, does not deny the existence of any of these achy, painful conditions, which are so common and so frequently demanding treatment. It does not deny that during damp weather, and in people who work or live in damp locations, there will be a distinct tendency to chronic aches and pains, just as there would be to frequent toothache under the same circumstances. It only transfers these affections from the column of chronic rheumatism, where they are all jumbled together, to the manifest opprobrium of our therapeutics, to separate columns, with regard to some of which we know our therapeutic impotency, but with regard to most of which we are fully aware that massage, rest, local treatment and improvement of the constitutional condition will do much to get rid of the symptoms. If the general public and the majority of the medical profession were once persuaded that chronic rheumatism, instead of being a common ailment, is, indeed, very rare, then there would be less failures of diagnosis, with consequent inevitable failures in treatment and less resort to our osteopathic friends, the enemy, and to the many remedies that are supposed to alter the uric acid diathesis and get rid of chronic rheumatic tendencies.

\section{DISCUSSION.}

Dr. JAmes Tyson, Philadelphia-Dr. Walsh has essayed a difficult subject, difficult as are all subjects characterized by an unknown or at least inexact pathology. There is confusion between gout and chronic or subacute rheumatism. While I 
think it is true that gout is a more frequent occurrence in America than has been supposed, I do feel sure that a great many cases are called gout without sufficient reason. I do not think a diagnosis of gout is justified unless there is or has been podagra, or uratic deposits in the joints or evident hereditary tendency. Heberden's nodosities, regarded as gouty by so many, may be gouty, but more frequently perhaps are a product of rheumatoid arthritis. I wish to urge on my fellow practitioners of medicine an attempt at more precise diagnosis of the two conditions. I am sure that in certain cases in my own experience many years have been required to determine whether a case be one of gout or rheumatism.

I should like to say a word also on the threadbare but still undetermined subject of the uric acid diathesis. I sympathize with Dr. Walsh in this respect, and it is mortifying to note how often rheumatic pains are ascribed to the uric acid diathesis without any reason whatever. It is often a careless expression. and not always intended by those who use it, but it gives an impression to the laity which is erroneous. As Dr. Walsh says, we really do not know what the uric acid diathesis is. There is, however, a clinical complex consisting in a scanty secretion of urine, of high specific gravity, with constant and copious deposits of uric acid and urates which is associated with the uric acid diathesis and is evidence of its presence. But it must be a permanent condition. It has doubtless happened to many of us to note in the morning after a liberal dinner including wines a scanty urine with uric acid deposits, etc., which will wear away after liberal ingestion of water, but this temporary state is not the uric acid diathesis. In the conditions referred to even the ingestion of large quantities of water does not increase the amount of urine secreted, and the tendency to sediments continues. To this condition I think we are justified in applying the name "uric acid diathesis." Such cases are not very numerous, and it may happen that associated muscular and joint pains are the result of it, but one ought at least to be sure of the combination before one asserts the causal relation.

Dr. Thomas MCCrae, Baltimore-Dr. Walsh has faced a question that the majority of us have faced frequently, and often with the diagnosis of "chronic rheumatism." The profession is largely to blame for the ideas the public have regarding this condition; we inherited the idea and pass it on. Dr. Walsh brought out a good point when he said that if one made a diagnosis of acute articular rheumatism and, after the attack is over, joint changes persist, then consider a wrong diagnosis; if we did that we would find fewer mistakes.

Dr. Alexander Lambert, New York-This helter-skelter diagnosis of rheumatism has been made for a long time, and my confrères in New York should take a more self-respecting attitude toward these painful affections. Often the diagnosis is easily made. It his for a long time struck me that so-called cases of chronic rheumatism with active tissue changes, ulnar deformities, etc., have nothing to do with rheumatism. It is perfectly true, in my opinion. that many cases began like articular rheumatism which can not be differentiated from it, which later prove to be true cases of arthritis deformans. In my experience this is seen most commonly in young women.

Grout is much more common in New York of late years, and it is certainly more often met with in hospital work than formerly. A great many cases that at first seem to be cases of rheumatism and yet which are treated as gout get well, and this is especially so in women where you get so often this irregular type of gout. That type of real chronic rheumatism is, as Dr. Walsh has stated, one that we must disregard. There are cases of rheumatism which begin as acute articular rheumatism, and before the patient recovers from one attack entirely they mav have another acute attack and then run on into a subacute condition that one must look on as chronic, a ehronic articular rheumatism. This process runs along a long while and leaves the patients more or less crippled. A great number of mistakes come from calling real gout rheumatism, and the ideas that are so prevalent regarding the uric acid diathesis have much to do with it. I am only too glad to be able to add my protest against this asylum for lazy diagnoses. I think it is firmly believed that the uric acid diathesis, occurring in healthy, vigorous, athletic, strong young men, is the cause of nearly all the woes they are heir to, especially the muscular pains. Whatever causes gout causes uric acid, and uric acid is simply one of the many symptoms. These men were usually 20 years old, and no one would accuse them of being gouty or rheumatic; they were in their prime and in vigorous health, and yet, among such, uric acid deposits in the urine are frequently found.

I wish to thank Dr. Walsh for bringing this subject up, because it is only by such papers and by such discussions can we realize that the so-called cases of gonorrheal rheumatism, scarlatinal rheumatism, etc., are not cases of rheumatism at all, but distinct and definite diseases; all cases naving pains in the joints should not be always considered as rheumatic, but they should be clearly differentiated. It is only by so doing that we will be able to be honest with ourselves and arrive at an accurate and scientific knowledge of the subject.

Dr. JAMES J. WALsir, New York-When I called my paper "The Passing of Chronic Rheumatism" I did not mean absolutely no cases of chronic rheumatism existed; I did mean to say that cases of chronic rheumatism were more or less curiosities and well worth studying. Chronic rheumatism is generally thought to be very common. If one should run across a case of true chronic rheumatism we would want a report of it in our journals. What is acute rheumatism? We think it is an acute infectious disease. A number of clinicians think the disease runs a course of from two to six weeks and gets better; they give salicylates, not because it is a specific for rheumatism, but because it lessens the pain and lowers the temperature. Again we have certain joint lestons occurring in gonorrhea, scarlet fever, typhoid fever and intestinal troubles that are accompanied by diarrheas, dysentery, etc., all of which may be accompanied by joint lesions and to which the term "rheumatism" is applied. I wish to enter a plea for a better knowledge of this condition.

It gives me pleasure to hear what Dr. Tyson has said regarding the uric acid diathesis; I think the uric acid diathesis has been overdone when we say that it gives rise to so many aches and pains, ete. If one looks into the literature one will find little authority for thinking the uric acid diathesis a prominent factor in disturbances of metabolism. We must remember that nervous peopie are more subject to pain than others; they may simply have some discomfort and think they really have pain, and such is particularly worse on rainy days. Dampness is associated with it.

\section{DANGERS ATTENDANT ON ATTEMPTS A'T GAINING THE INTERVAL IN OPERA- TIONS FOR APPENDICITIS.* RICHAKD H. GIBBONS, M.D. SCRANTON.}

It is strange that medical men in general and anatomists and physiologists in particular claim that the appendix vermiformis has no function, that it is a useless appendage, playing the part of catching any old thing, or furnishing the surgeon opportunity to rake in illgotten gain. Ridiculous as it is to compare inanimate things to parts of the human body, it may be well to do so here, in order to state that at such a right-angled junction as is formed by the ileocecal connection, an oil cup would be furnished in mechanies, while in hydraulics, as in a sewerage system, for instance, a manhole would be placed. This appendage apparently has secretory functions by which, it is fair to assume, the natural tendency to pastiness of the residue of digestion, after it comes to the cecum, is so modified that it is ren-

* Read at the Fifty-fourth Annual Session of the American Medical Association, in the Section on Surgery and Anatomy, an approved for publication by the Executive Committee: Drs. A. J. approved for publication by the Executive Cond
Ochsner, J. E. Moore and De Forest Willard. 